\title{
Perfil sociodemográfico e de eliminações intestinais em mulheres atendidas em um serviço de saúde
}

\section{Perfil sociodemográfico y de eliminación intestinal en mujeres tratadas en un senvicio de salud}

Socio-demographic and intestinal elimination profile in women treated at a health service

Rhaylla Maria Pio Leal Jaques, ,*, Elaine Carininy Lopes da Costa², Gisela Maria Assis³, Aline Costa de Oliveira ${ }^{4}$, Claudia Daniella Avelino Vasconcelos Benício5, Sandra Marina Gonçalves Bezerra ${ }^{4}$

ORCID IDS

Jaques RMPL (D) https://orcid.org/ 0000-0002-2250-3886

Costa ECL (D) https://orcid.org/0000-0002-5387-978X

Assis GM (iD https://orcid.org/ 0000-0001-6343-8075

Oliveira AC (D) https://orcid.org/ 0000-0003-1738-4808

Benício CDAV (iD https://orcid.org/ 0000-0003-4638-2465

Bezerra SMG (D) https://orcid.org/ 0000-0003-3890-5887

\section{COMO CITAR}

Jaques RMPL; Costa ECL; Assis GM; Oliveira AC; Benício CDAV; Bezerra SMG. Perfil sociodemográfico e de eliminações intestinais em mulheres atendidas em um serviço de saúde. ESTIMA, Braz. J. Enterostomal Ther., 18: e0820, 2020. https://doi.org/10.30886/ estima.v18.830_PT

\section{RESUMO}

Objetivo: Investigar o perfil de eliminações intestinais em mulheres. Método: Estudo transversal, realizado com 41 mulheres atendidas em um centro de saúde da mulher no estado do Piauí no Brasil. Para coleta de dados foi utilizado um formulário contendo os dados sociodemográficos e questionários que avaliaram os dados do padrão de eliminação intestinal com perguntas fundamentadas nos critérios de Roma IV e escala de Bristol. Resultados: Das 41 participantes, 56,1\% relataram consumir de uma a duas porções de frutas ou verduras por dia e 51,2\% consumiam mais de dois litros de água por dia. Com relação aos padrões de eliminação intestinal, 39\% apresentavam fezes na categoria Bristol 3. Quanto aos critérios de Roma IV, 21 (51,2\%) mulheres foram consideradas constipadas e a frequência de evacuação teve significância estatística com a presença de constipação. Conclusão: Houve um número expressivo de mulheres com constipação intestinal. Observou-se a necessidade de desenvolver ações que auxiliem no enfrentamento do problema e melhore a qualidade de vida dessa população.

DESCRITORES: Constipação intestinal. Perfil Epidemiológico. Saúde da mulher. Estomaterapia.

\begin{abstract}
Objective: To investigate the profile of intestinal eliminations in women. Method: Cross-sectional study conducted with 41 women seen at a women's health center in the state of Piauí in Brazil. For data collection, a form was used containing the sociodemographic data and questionnaires that assessed the data on the intestinal elimination pattern with questions based on the Rome IV criteria and the Bristol scale. Results: Of the 41 participants, 56.1\% reported consuming one to two servings of fruits or vegetables per day and $51.2 \%$ consumed more than two liters of water per day. Regarding the patterns of intestinal elimination, 39\% had feces in the Bristol 3 category. As for the Rome IV criteria, 21 (51.2\%) women were considered constipated and the frequency of evacuation was statistically significant with the presence of constipation. Conclusion: There was a significant number of women with constipation. There was a need to develop actions that help to cope with the problem and improve the quality of life of this population.
\end{abstract}

DESCRIPTORS: Constipation. Health profile. Women's health. Enterostomal therapy.

\footnotetext{
1.Clínica Integrada de Saúde da Mulher - Picos (PI), Brasil

2.Instituto Federal do Piauí - Setor de Saúde - Oeiras (PI), Brasil

3.Universidade Federal do Paraná - Ambulatório de Incontinência do Hospital das Clínicas - Curitiba (PR), Brasil

4.Universidade Estadual do Piauí - Coordenação de Enfermagem em Estomaterapia - Teresina (PI), Brasil

5.Universidade Federal do Piauí - Coordenação de Enfermagem - Teresina (PI), Brasil
}

*Autora correspondente: rhaylla.maria@hotmail.com

Recebido: Nov. 28, 2019 | Aceito: Mar. 27, 2020 


\section{RESUMEN}

Objetivo: Investigar el perfil de eliminaciones intestinales en mujeres. Método: Estudio transversal realizado con 41 mujeres atendidas en un centro de salud para mujeres en el estado de Piauí en Brasil. Para la recolección de datos, se utilizó un formulario que contenía los datos sociodemográficos y los cuestionarios que evaluaban los datos sobre el patrón de eliminación intestinal con preguntas basadas en los criterios de Roma IV y la escala de Bristol. Resultados: De los 41 participantes, el 56.1\% informó consumir una o dos porciones de frutas o verduras por día y el 51.2\% consumió más de dos litros de agua por día. Con respecto a los patrones de eliminación intestinal, el 39\% tenía heces en la categoría de Bristol 3. En cuanto a los criterios de Roma IV, 21 (51,2\%) mujeres se consideraron estreñidas y la frecuencia de evacuación fue estadísticamente significativa con la presencia de estreñimiento. Conclusión: Hubo un número significativo de mujeres con estreñimiento y fue necesario desarrollar acciones que ayuden a enfrentar el problema y mejorar la calidad de vida de esta población.

DESCRIPTORES: Estreñimiento. Perfil de salud. Salud de la mujer. Estomaterapia..

\section{INTRODUÇÃO}

O padrão de eliminação intestinal pode influenciar na qualidade de vida do indivíduo, sobretudo quando ocorrem alterações nos padrões normais de eliminação. Dentre as alterações, destaca-se a constipação intestinal (CI), que pode influenciar o cotidiano das pessoas ${ }^{1}$.Embora essas manifestações não sejam consideradas problemas de saúde pública, elas se tornam relevantes pois podem desencadear, em alguns casos, reações que favoreçam o isolamento social e a baixa autoestima prejudicando assim a qualidade de vida do indivíduo ${ }^{1}$.

A CI é definida como defecação insatisfatória, caracterizada por dificuldade na passagem das fezes, fezes infrequentes, fezes duras ou sensação de evacuação incompleta, ocorrendo de forma isolada ou associada a uma doença ${ }^{2}$. Embora a CI não seja uma ameaça à vida do indivíduo, possui um efeito negativo na qualidade de vida ${ }^{3}$.

Para concluir o diagnóstico de CI, é necessário que o indivíduo apresente, no mínimo durante três meses, dois ou mais dos seguintes sintomas, elencados nos critérios de Roma IV: fezes endurecidas, frequência de evacuação menor que três vezes por semana, esforço ao defecar, necessidade de realizar as manobras manuais, sensação de evacuações incompletas em pelo menos $25 \%$ das vezes, e/ou sensação de bloqueio/obstrução anorretal das fezes ${ }^{3,4}$.

A CI é multifatorial, podendo estar associada a alterações sistêmicas ou neurológicas, bem como ao uso de medicamentos e dietas inadequadas com baixo consumo de fibras, idade, sexo feminino, baixo nível socioeconômico, baixa escolaridade, disfunções endócrinas e metabólicas, inatividade física, distúrbios psiquiátricos e causas idiopáticas. Além disso, a CI pode ser um sintoma inicial de doenças graves, como o câncer colorretal 5 .
Com relação ao manejo da CI, algumas estratégias podem ser estimuladas e orientadas pelo enfermeiro, em especial o estomaterapeuta, tais como dieta rica em fibras, aumento da ingestão de líquidos, realização de atividade física, utilização de banco para melhorar a postura no momento da evacuação, sentar no vaso sanitário após as refeições de preferência sempre no mesmo horário para estabelecer a rotina (aproveitando o aumento do reflexo gastrocólico), realização da manobra de Valsalva (que consiste em inspirar profundamente e forçar os músculos do abdômen e diafragma para baixo) bem como massagens abdominais (massageando o cólon da direita para esquerda, no sentido de cima para baixo, durante vinte minutos $)^{6,7}$.

A escassez de informações oficiais considerando o padrão de eliminação intestinal e urinárias em mulheres pode prejudicar o planejamento e a implementação de estratégias e alocação de recursos para a melhoria da assistência de enfermagem. Nesse sentido, a obtenção dessas informações visa o melhor atendimento ao cliente, ampliando o papel e o compromisso profissional do enfermeiro desde a prevenção até o processo de reabilitação, aplicando no cuidar a mediação da educação na busca da autonomia para melhoria da qualidade de vida dessa clientela.

Este estudo teve o objetivo de investigar o perfil sociodemografico e de eliminações intestinais em mulheres atendidas em um centro de referência à saúde da mulher.

\section{MÉTODO}

Trata-se de uma pesquisa transversal, descritiva com abordagem quantitativa. $\mathrm{O}$ estudo foi realizado em um Centro de Saúde da Mulher, no estado do Piauí no Brasil. 
A população do estudo foi constituída por mulheres que estavam aguardando a consulta na sala de espera do serviço. A amostra foi do tipo não probabilística, por conveniência e composta por 41 mulheres atendidas no serviço durante o período de coleta de dados. Foram incluídas aquelas mulheres com idade superior a 18 anos.

A coleta de dados foi realizada nos meses de junho e julho de 2019 pela pesquisadora responsável pelo estudo. As mulheres foram abordadas individualmente na sala de espera, sendo informadas pela pesquisadora sobre a pesquisa; aquelas que concordaram em participar foram encaminhadas para um consultório, onde o questionário foi preenchido com garantia de privacidade.

Os instrumentos de coleta de dados foram constituídos por um formulário produzido pela pesquisadora para esta pesquisa, contendo os dados sociodemográficos e os questionários que avaliaram os dados do padrão de eliminação intestinal com perguntas fundamentadas nos critérios de Roma $\mathrm{IV}^{4}$ e escala de Bristol ${ }^{8}$.

Os critérios de Roma constitui uma diretriz recente desenvolvida para padronizar o diagnóstico e o manejo da constipação intestinal. Se o participante apresentar, durante um periodo mínimo de três meses, dois ou mais dos seguintes critérios: fezes endurecidas, menos de três movimentos intestinais espontâneos por semana, esforço ao defecar, manobras manuais, sensação de evacuações incompletas, sensação de bloqueio/obstrução anorretal das fezes, confirma-se o diagnóstico de $\mathrm{CI}^{4}$.

A escala de Bristol tem o objetivo de avaliar e descrever a forma do conteúdo fecal, utilizando métodos gráficos que representam sete tipos de fezes, de acordo com sua forma e consistência, sendo útil a pacientes para avaliarem e descreverem aspectos de suas fezes, facilitando o reconhecimento da gravidade da constipação ${ }^{3}$. O tipos de fezes descritos no instrumento são: tipo 1 (pequenas bolinhas duras, separadas como coquinhos - dificil para sair), tipo 2 (formato de linguiça encaroçada, com pequenas bolinhas grudadas), tipo 3 (formato de linguiça com rachaduras na superfície), tipo 4 (alongada com formato de salsicha ou cobra, lisa e macia), tipo 5 (pedaços maiores e separados com bordas bem definidas - fáceis de sair), tipo 6 (massa pastosa e fofa, com bordas irregulares), tipo 7 (totalmente líquidas, sem pedaços sólidos) $)^{8}$.

Os dados foram digitados no software Microsoft Excel e analisados com a utilização do software Statistical
Package for Social Science (SPSS) versão 22.0, ferramenta de tratamento de dados e análise estatística. Foram realizadas análises descritivas, como medidas de tendência central para as variáveis numéricas (média e mediana), frequência absoluta e percentual para as variáveis qualitativas e medidas de dispersão ou variabilidade (desvio padrão). Para a análise inferencial, foi utilizado o teste exato de Fisher, considerando estatisticamente significantes os resultados que apresentaram valor $p$ menor ou igual a 0,05 .

Foram seguidas todas as recomendações da Resolução 466/2012 do Conselho Nacional de Saúde (CNS) e o projeto foi aprovado pelo Comitê de Ética em Pesquisa da Universidade Estadual do Piauí com seres humanos sob parecer de n. 3.447.450.

\section{RESULTADOS}

Das 41 participantes da pesquisa $11(26,8 \%)$ estavam na faixa etária entre 30 a 39 anos, $13(31,7 \%)$ possuíam o ensino fundamental completo e $24(58,5 \%)$ eram casadas. Quanto à ocupação profissional, 13 (31,7\%) eram lavradoras. Quinze (36,6\%) das participantes não possuíam doença associada. Houve predomínio de $40(97,6 \%)$ mulheres não fumantes e $35(85,4 \%)$ não consumiam bebidas alcoólicas, conforme apresentado na Tabela 1.

Durante a investigação dos fatores de risco para constipação intestinal (CI), 24 (58,5\%) mulheres relataram que urinavam na cama ao dormir e $19(46,4 \%)$ não tinham perda de urina durante período da infância, porém 18 $(43,9 \%)$ não lembravam ter tido constipação nesse período. Cerca de $21(51,2 \%)$ mulheres investigadas referiram boa ingestão de água, $23(56,1 \%)$ tinham baixa ingestão de fibras, sendo predominante o consumo de vegetais de uma a duas vezes por dia e $25(61,1 \%)$ tinham frequência de evacuação diária. Quanto à autopercepção de ansiedade, 32 (78\%) participantes percebem-se como ansiosas (Tabela 2).

Ao investigar as variáveis relacionadas à eliminação intestinal, percebeu-se que o tipo de fezes mais comum, de acordo com a escala de Bristol, foi do tipo 3, presente em 16 (39\%) participantes, seguido do tipo 4 em 11 $(26,8 \%)$ pessoas.

Considerando como CI a presença de dois ou mais sintomas dos critérios de Roma IV, 21 (51,2\%) 
mulheres eram constipadas. Na Tabela 3 são apresentadas a frequência e o percentual de cada um dos sintomas investigados pelos critério de Roma.

$\mathrm{Na}$ Tabela 4, observou-se que a frequência de evacuação teve associação estatística com a variável constipação intestinal ( $\mathrm{p}=0,007)$, na qual é possível verificar que $11(52,4 \%)$ pacientes consideradas constipadas tinham frequência evacuatória de apenas 2 a 4 vezes na semana.

Tabela 1. Descrição das características sociodemográficas das participantes do estudo. Picos - Piauí - 2019.

\begin{tabular}{|c|c|c|c|}
\hline Características & Categorias & $\mathrm{n}=41$ & $\%$ \\
\hline \multirow{5}{*}{ Faixa etária } & De 18 a 29 anos & 6 & 14,6 \\
\hline & De 30 a 39 anos & 11 & 26,8 \\
\hline & De 40 a 49 anos & 8 & 19,5 \\
\hline & De 50 a 59 anos & 7 & 17,1 \\
\hline & Mais de 60 anos & 9 & 22,0 \\
\hline \multirow{7}{*}{$\begin{array}{c}\text { Grau de } \\
\text { escolaridade }\end{array}$} & Analfabeto & 3 & 7,3 \\
\hline & Fundamental incompleto & 4 & 9,8 \\
\hline & Fundamental completo & 13 & 31,7 \\
\hline & Médio incompleto & 5 & 12,2 \\
\hline & Médio completo & 8 & 19,5 \\
\hline & Superior & 7 & 17,1 \\
\hline & Superior incompleto & 1 & 2,4 \\
\hline \multirow{5}{*}{ Estado civil } & Solteira & 4 & 9,8 \\
\hline & Casada & 24 & 58,5 \\
\hline & Viúva & 2 & 4,9 \\
\hline & Separada & 5 & 12,2 \\
\hline & União estável & 6 & 14,6 \\
\hline \multirow{7}{*}{$\begin{array}{c}\text { Ocupação } \\
\text { profissional }\end{array}$} & Aposentada & 3 & 7,3 \\
\hline & Lavradora & 13 & 31,7 \\
\hline & Outra & 12 & 29,3 \\
\hline & Estudante & 2 & 4,9 \\
\hline & Microempreendedora & 1 & 2,4 \\
\hline & Professora & 3 & 7,3 \\
\hline & Dona de casa & 7 & 17,1 \\
\hline \multirow{5}{*}{$\begin{array}{c}\text { Doença } \\
\text { associada }\end{array}$} & Hipertensão & 11 & 26,8 \\
\hline & Anemia & 3 & 7,3 \\
\hline & Outra & 7 & 17,1 \\
\hline & Não & 15 & 36,6 \\
\hline & Mais de uma opção & 5 & 12,2 \\
\hline \multirow{2}{*}{ Fuma } & Sim & 1 & 2,4 \\
\hline & Não & 40 & 97,6 \\
\hline \multirow{2}{*}{ Consome álcool } & Sim & 6 & 14,6 \\
\hline & Não & 35 & 85,4 \\
\hline
\end{tabular}

Tabela 2. Descrição das características clínicas das participantes do estudo. Picos - Piauí - 2019.

\begin{tabular}{|c|c|c|c|}
\hline Característica & Categoria & $\mathrm{n}=41$ & $\%$ \\
\hline \multirow{3}{*}{$\begin{array}{l}\text { Urinava na cama } \\
\text { ao dormir }\end{array}$} & Sim & 24 & 58,5 \\
\hline & Não & 12 & 29,3 \\
\hline & Não lembra & 5 & 12,2 \\
\hline \multirow{3}{*}{$\begin{array}{c}\text { Perda de urina na } \\
\text { infância }\end{array}$} & $\operatorname{Sim}$ & 6 & 14,6 \\
\hline & Não & 19 & 46,4 \\
\hline & Não lembra & 16 & 39,0 \\
\hline \multirow{4}{*}{$\begin{array}{c}\text { Constipação } \\
\text { intestinal na } \\
\text { infância }\end{array}$} & $\mathrm{Sim}$ & 6 & 14,6 \\
\hline & Não & 17 & 41,5 \\
\hline & Não lembra & 18 & 43,9 \\
\hline & De 1 a 3 copos/dia & 4 & 9,8 \\
\hline \multirow{3}{*}{$\begin{array}{c}\text { Consome quantos } \\
\text { copos de água? }\end{array}$} & De 4 a 6 copos/dia & 11 & 26,8 \\
\hline & De 7 a 9 copos/dia & 5 & 12,2 \\
\hline & Mais de 10 copos/dia & 21 & 51,2 \\
\hline \multirow{4}{*}{$\begin{array}{c}\text { Consome frutas e } \\
\text { verduras? }\end{array}$} & Nenhuma & 6 & 14,6 \\
\hline & De 1 a 2 vezes/dia & 23 & 56,1 \\
\hline & De 3 a 5 vezes/dia & 12 & 29,3 \\
\hline & Todo dia & 25 & 61,1 \\
\hline \multirow{3}{*}{$\begin{array}{l}\text { Frequência de } \\
\text { evacuação }\end{array}$} & 1 vez na semana & 1 & 2,4 \\
\hline & De 2 a 4 vezes/semana & 14 & 34,1 \\
\hline & De 10 em 10 dias & 1 & 2,4 \\
\hline \multirow{3}{*}{$\begin{array}{l}\text { Ansiedade e/ou } \\
\text { depressão }\end{array}$} & Ansiosa & 32 & 78,0 \\
\hline & Não se aplica & 7 & 17,1 \\
\hline & Ambos & 2 & 4,9 \\
\hline
\end{tabular}

Tabela 3. Descrição das características de eliminação intestinal das participantes do estudo, segundo a escala de Bristol e os critérios de Roma IV. Picos - Piauí - 2019.

\begin{tabular}{|c|c|c|c|}
\hline Característica & Categoria & $n=41$ & $\%$ \\
\hline \multirow{7}{*}{ Escala de Bristo|* } & Bristol 1 & 7 & 17,1 \\
\hline & Bristol 2 & 5 & 12,2 \\
\hline & Bristol 3 & 16 & 39,0 \\
\hline & Bristol 4 & 11 & 26,8 \\
\hline & Bristol 5 & 5 & 12,2 \\
\hline & Bristol 6 & 2 & 4,9 \\
\hline & Bristol 7 & 1 & 2,4 \\
\hline \multicolumn{4}{|l|}{ Critérios de Roma III* } \\
\hline \multicolumn{2}{|l|}{ Esforço para evacuar } & 17 & 41,5 \\
\hline \multicolumn{2}{|c|}{ Sensação de evacuação incompleta } & 16 & 39,0 \\
\hline \multicolumn{2}{|c|}{ Realiza manobras manuais } & 7 & 17,1 \\
\hline \multicolumn{2}{|c|}{ Fezes grumosas ou endurecidas } & 20 & 48,8 \\
\hline \multicolumn{2}{|c|}{$\begin{array}{l}\text { Sensação de obstrução/bloqueio } \\
\text { anorretal por fezes }\end{array}$} & 13 & 31,7 \\
\hline \multicolumn{2}{|c|}{ Evacua menos de três vezes por semana } & 6 & 14,6 \\
\hline \multicolumn{2}{|c|}{ Diagnostico de constipação } & 21 & 51,2 \\
\hline
\end{tabular}

*A participante poderia indicar mais de uma opção, assim sendo, o total de frequência e percentual não somam 41 e 100\%, respectivamente. 
Tabela 4. Comparação entre mulheres constipadas e não constipadas e as características clínicas referentes à eliminação intestinal. Picos - Piauí - 2019.

\begin{tabular}{|c|c|c|c|c|}
\hline \multirow{2}{*}{ Característica } & \multirow{2}{*}{ Categoria } & \multirow{2}{*}{$\begin{array}{c}\text { Constipadas } \\
\text { n (\%) }\end{array}$} & \multirow{2}{*}{$\begin{array}{c}\text { Não constipadas } \\
\text { n (\%) }\end{array}$} & \multirow{2}{*}{ Valor $\mathrm{p}$} \\
\hline & & & & \\
\hline \multirow{3}{*}{$\begin{array}{c}\text { Constipação intestinal } \\
\text { na infância }\end{array}$} & Sim & $4(19,0)$ & $2(10,0)$ & \multirow{3}{*}{$0,708^{*}$} \\
\hline & Não & $9(42,9)$ & $8(40,0)$ & \\
\hline & Não lembra & $8(38,1)$ & $10(50,0)$ & \\
\hline \multirow{4}{*}{$\begin{array}{l}\text { Consome quantos } \\
\text { copos de água? }\end{array}$} & De 1 a 3 copos/dia & $2(9,5)$ & $2(10,0)$ & \multirow{4}{*}{$0,860 *$} \\
\hline & De 4 a 6 copos/dia & $7(33,3)$ & $4(20,0)$ & \\
\hline & De 7 a 9 copos/dia & $2(9,5)$ & $3(15,0)$ & \\
\hline & Mais de 10 copos/dia & $10(47,6)$ & $11(55,0)$ & \\
\hline \multirow{3}{*}{$\begin{array}{c}\text { Consome frutas e } \\
\text { verduras? }\end{array}$} & Nenhuma & $3(14,3)$ & $3(15,0)$ & \multirow{3}{*}{$0,072 *$} \\
\hline & De 1 a 2 vezes/dia & $15(71,4)$ & $8(40,0)$ & \\
\hline & De 3 a 5 vezes/dia & $3(14,3)$ & $9(45,0)$ & \\
\hline \multirow{4}{*}{$\begin{array}{c}\text { Frequência de } \\
\text { evacuação }\end{array}$} & Todo dia & $8(38,1)$ & $17(85,0)$ & \multirow{4}{*}{$0,007 *$} \\
\hline & 1 vez na semana & $1(4,8)$ & $0(0,0)$ & \\
\hline & De 2 a 4 vezes/semana & $11(52,4)$ & $3(15,0)$ & \\
\hline & De 10 em 10 dias & $1(4,8)$ & $0(0,0)$ & \\
\hline \multirow{3}{*}{$\begin{array}{c}\text { Ansiedade e/ou } \\
\text { depressão }\end{array}$} & Ansiosa & $17(81,0)$ & $15(75,0)$ & \multirow{3}{*}{$0,836 *$} \\
\hline & Não se aplica & $3(14,3)$ & $4(20,0)$ & \\
\hline & Os dois & $1(4,8)$ & $1(5,0)$ & \\
\hline
\end{tabular}

* Teste exato de Fisher

\section{DISCUSSÃO}

Este estudo contou com a participação de mulheres na faixa etária de 18 a 78 anos, sendo a faixa mais prevalente de 30 a 39 anos, embora a literatura aponte prevalência de CI em mulheres na faixa etária de 40 e 59 anos $^{8}$.

Com relação à investigação do grau de escolaridade das participantes, foi possível perceber baixo nível de escolaridade com predominio de casadas como estado civil casadas ${ }^{9,10}$. A literatura também aponta maior prevalência de CI no sexo feminino em decorrência das alterações que ocorrem no assoalho pélvico por ocasião da gestação e do parto 5 . Além disso, as questões hormonais também são apontadas como fator de risco de CI, como a ação da progesterona que se encontra elevada na fase lútea do ciclo menstrual e durante o segundo e terceiro trimestres da gestação, bem como os danos à musculatura do assoalho pélvico durante cirurgias ginecológicas e obstétricas que podem aumentar a incidência ${ }^{11}$.

Quanto à ocorrência de doenças associadas, 26,8\% das participantes referiu hipertensão arterial. Tal resultado confirma aqueles encontrados em pesquisa realizada no Nordeste brasileiro sobre CI em que aponta prevalência semelhante de pessoas com hipertensão arterial como comorbidade presente entre os investigados ${ }^{12}$. Ao investigar o tabagismo entre as participantes, observou-se que a maioria relatou não fumar. Apesar de questionável, a literatura aponta que o hábito de fumar é inversamente proporcional à CI; tal ocorrência está associada ao fato de que a nicotina é capaz de facilitar o esvaziamento do intestino grosso por relaxar o esfíncter anal, o que vai influenciar no movimento intestinal ${ }^{13}$.

Quanto aos fatores de risco para a ocorrência de CI, observou-se que a maioria das mulheres investigadas $(21 / 51,2 \%)$ consumia mais de 10 copos $(200 \mathrm{ml})$ de água por dia. É importante ressaltar que a ingestão de líquidos, incluindo sucos e água, deve ser de pelo menos oito copos/dia pois a hidratação adequada altera a consistência e o peso das fezes, aumenta o número de reflexos gastrocólicos e contribui para a lubrificação intestinal $^{14}$.

Com relação ao consumo de frutas e verduras, foi evidenciado neste estudo que a maioria consumia de uma a duas porções de frutas e verduras por dia, coincidindo com estudo realizado em Portugal que abordou 202 pessoas e evidenciou que mais $50 \%$ da população consumiam frutas e verduras diariamente ${ }^{4}$. Em contrapartida, um 
estudo evidenciou que a maioria dos participantes nem sempre consumiam frutas e verduras ${ }^{1}$. As fibras alimentares presentes nas frutas e verduras são componentes não digeridos pelas enzimas digestivas do trato gastrintestinal dos seres humanos, dividindo-se em solúveis que colaboram para a maciez das fezes e insolúveis que aumentam o volume fecal, estimulando as ondas peristálticas e o esvaziamento do cólon ${ }^{15}$.

Quanto à frequência de evacuação, a maioria das participantes relatou que evacua todos os dias. Resultado diferente foi encontrado em estudos em que houve predomínio de participantes que evacuavam apenas uma vez na semana ${ }^{11}$. Este resultado reforça a necessidade de se ampliar o conceito de constipação, pois, considerando apenas a frequência evacuatória, a minoria pareceria constipada, porém, com a aplicação dos critérios de Roma, os sintomas apontaram para outros aspectos de evacuação insatisfatória.

No que diz respeito ao comportamento emocional, a maior parte das participantes relatou sofrer de ansiedade. É importante considerar a associação entre a $\mathrm{CI}$ e condições psicológicas tais como estresse, ansiedade e depressão, pois essas manifestações predispõem à CI, com possível implicação na etiologia da doença ${ }^{11}$.

Ao investigar o tipo de fezes segundo a escala de Bristol, percebeu-se maior frequência de fezes tipo 3, corroborando os resultados de diversos estudos ${ }^{4,16,17}$. Com relação à consistência ideal das fezes, a literatura aponta que as fezes classificadas como tipo 4 são as mais adequadas, caracterizando-se como fezes alongadas, em formato de salsicha ou cobra, lisa e macia ${ }^{8}$.

O indivíduo, para ter o diagnóstico de constipação intestinal, deve apresentar pelo menos duas ou mais manifestações dos critérios de Roma IV, no período de três meses. Neste estudo, observou-se que a maioria das participantes tinham constipação intestinal ${ }^{4,17}$, com predomínio de fezes grumosas ou endurecidas e sensação de esvaziamento incompleto. Tal resultado também pode ser encontrado em diversos estudos sobre a prevalência da constipação intestinal ${ }^{18,19}$.

Vale ressaltar que o tamanho amostral foi uma limitação do estudo, imposta pelo tempo disponível para a coleta de dados. Essa limitação pode ser a justificativa de não ter havido associação entre o diagnóstico de constipação intestinal e fatores de risco amplamente conhecidos. Apesar disso, chama atenção o número de mulheres constipadas que se descrevem como ansiosas.

\section{CONCLUSÃO}

O estudo demonstrou uma presença expressiva de constipação intestinal entre as mulheres atendidas em um centro de atenção à saúde da mulher, mesmo que esse não tenha sido o motivo para terem buscado o serviço. Embora a relação entre variáveis não tenha sido comprovada estatisticamente, é relevante o número de mulheres que recordavam ter vivenciado enurese na infância e que se consideram ansiosas.

O resultado aponta para uma necessidade de se trabalhar o tema entre grupos de mulheres, sabendo-se que é um grupo mais susceptível ao problema. Desperta também para a possibilidade de atuação do enfermeiro com ações de educação em saúde, tanto no sentido de trazer o tema para a discussão, quanto na ação direta para prevenção e tratamento, seja em consultas individuais, ações em grupo ou campanhas de orientação.

Sugere-se a realização de outras pesquisas envolvendo a temática da constipação intestinal em mulheres, possibilitando o conhecimento da dimensão real do problema, bem como a avaliação do resultado de ações de prevenção e tratamento, de forma a promover a inserção do tema na atenção primária e secundária de saúde, envolvendo o enfermeiro como agente de mudança e promotor de qualidade de vida, nessa área ainda tão negligenciada.

\section{AGRADECIMENTOS}

Às mulheres participantes da pesquisa, Coordenação da Pós Graduação em Estomaterapia, Professores e Enfermeiras da Primeira Turma de Estomaterapia da Universidade Estadual do Piauí.

\section{CONTRIBUIÇÃO DOS AUTORES}

\section{Conceitualização, Jaques RMPL, Assis GM e Oliveira} AC; Investigação,Jaques RMPL; Redação - Primeira versão, Jaques RMPL, CostaECL, Assis GM, Oliveira AC, Benício CDAV e Bezerra SMG; Redação - Revisão \&Edição, Jaques RMPL, CostaECL, Assis GM, Oliveira AC, Benício CDAV e Bezerra SMG; Supervisão, Jaques RMPL. 


\section{REFERÊNCIAS}

1. Bomfim IQ, Nunes LS, Alves TC. Prevalência de constipação intestinal em estudantes de fisioterapia de uma Universidade de Maceió/AL. Rev Ciênc Méd Biol. 2017;16(1):79-84. https:// doi.org/10.9771/cmbio.v16i1.13266

2. Schmidt FMQ, Santos VLCG, Domansky RC, Barros E, Bandeira MA, Tenório MAM, et al. Prevalência de constipação intestinal autorreferida em adultos da população geral. Rev Esc Enferm USP. 2015;49(3):443-52. https://doi. org/10.1590/S0080-623420150000300012

3. Sobrado CW, Corrêa Neto IJF, Pinto RA, Sobrado LF, Nahas SC, Cecconello I. Diagnosis and treatment of constipation: a clinical update based on the Rome IV criteria. J Coloproctol. 2018;38(2):137-44. https://doi.org/10.1016/j. jcol.2018.02.003

4. Azevedo R, Ribeiro H, Pinto J, Leitão C, Caldeira A, Banhudo A. Modelo preditivo de obstipação: o que poderá ser útil para além do Roma IV? Rev Port Coloproct. 2017;14(2):12-9.

5. Garcia LB, Bertolini SMMG, Souza MV, Santos MSF, Pereira COM. Constipação intestinal: aspectos epidemiológicos e clínicos. Saúde Pesqui. 2016;9(1):153-62. https://doi. org/10.17765/1983-1870.2016v9n1p153-162

6. Machado D, Assis GM. Padrão intestinal e medidas de controle adotadas por pacientes com trauma raquimedular. ESTIMA, BrazJ Enterostomal Ther. 2018;16:e1418.

7. Braz MM, Kelling BI, Arruda GT, Stallbaum JH. A constipação intestinal em idosas participantes de um programa de promoção à saúde, em Santa Maria (RS): sua prevalência, sintomas e fatores psicossociais associados. Rev Kairós. 2015;18(3):381-95.

8. Martinez AP, Azevedo GR. Tradução, adaptação cultural e validação da Bristol Stool Form Scale para a população brasileira. Rev Latino-Am Enfermagem. 2012;20(3):1-7. https://doi.org/10.1590/S0104-11692012000300021

9. Cunha RM, Leite LC, Oliveira GND, Brito GA, Figueredo VB, Nascimento SL. Perfil epidemiológico e sintomas urinários de mulheres com disfunções do assoalho pélvico atendidas em ambulatório. Rev Fisioter S Fun. 2016;5(1):42-9.

10. Lopes MHBM, Costa JN, Bicalho MB, Casale TE, Camisão AR, Fernandes MLV. Profile and quality of life of women in pelvic floor rehabilitation. Rev Bras Enferm. 2018;71(5):2642-51. https://doi.org/10.1590/0034-7167-2017-0602

11. Trisógilio C, Marchi CMG, Torres US, Netinho JG. Prevalência de constipação intestinal entre estudantes de medicina de uma instituição no Noroeste Paulista. Rev Bras Coloproct. 2010;30(2):203-9. https://doi.org/10.1590/S010198802010000200012

12. Silva MS, Pinho CPS. Constipação intestinal: prevalência e fatores associados em pacientes atendidos ambulatorialmente em hospital do Nordeste brasileiro. Nutr Clín Diet Hosp. 2016;36(1):75-84. https://doi. org/10.12873/361sousa

13. Jaime RP, Campos RC, Santos TST, Marques MS. Prevalência e fatores de risco da constipação intestinal em universitários de uma instituição particular de Goiânia, GO. Rev Inst Ciênc Saúde. 2009;27(4):378-83.

14. Gavanski DS, Baratto I, Gatti RR. Avaliação do hábito intestinal e ingestão de fibras alimentares em uma população de idosos. Rev Bras Obes Nutr Emagrecimento. 2015;9(49):3-11.

15. Muñoz RLS, Santos LA, Martins MMC, Araújo DU, Vieira ATP, Vilar GN, et al. Constipação intestinal e fatores associados em estudantes universitários da área de saúde. Rev Salusvita (Online). 2016;35(3):351-66.

16. Dall Agnol TM, Araújo MP, Laino F, Parmigiano TR, Girão MJBC, Sartori MGF. Avaliação do habito intestinal em mulheres atletas e sua relação com nível de hidratação e uso de suplemento. Rev Bras Nutr Esportiva. 2016;10(58):458-66.

17. Pinheiro AK, Geron VLMG, Terra Júnior AT, Nunes JS, Brondani FMM. Constipação intestinal: tratamento com fitoterápicos. Rev Cient Fac Educ e Meio Ambient. 2018;9(ed esp):559-64. https://doi.org/10.31072/rcf.v9iedesp.598

18. Olivon EV, Costa JJ, Machado AD, Chaud DMA, Abreu ES. Prevalência e fatores associados à constipação intestinal funcional em universitários. Ciência e Saúde. 2016;9(3):1505. https://doi.org/10.15448/1983-652X.2016.3.22591

19. Sant'Anna MSL, Ferreira CLLF. Prevalência de constipação intestinal no município de Viçosa/MG. Nutrição Brasil. 2016;15(1):10-4. 